\title{
Effect of step-edge on spectral properties and planar stability of metallic bigraphene
}

\author{
V.V. Eremenko, V.A. Sirenko, I.A. Gospodarev, E.S. Syrkin, \\ S.B. Feodosyev, and I.S. Bondar \\ B. Verkin Institute for Low Temperature Physics and Engineering of the National Academy of Sciences of Ukraine \\ 47 Pr. Nauky, Kharkiv 61103, Ukraine \\ E-mail: feodosiev@ilt.kharkov.ua
}

S.S. Saxena

Cavendish Laboratory, University of Cambridge, JJ Thomson Avenue, Cambridge CB3 OHE, United Kingdom

\begin{abstract}
A. Feher
Centre of Low Temperature Physics, Faculty of Science, P.J. Šafárik University and Institute of Experimental Physics SAS, 9 Park Angelinum, Košice 041 54, Slovakia
\end{abstract}

K.A. Minakova

National Technical University “Kharkiv Polytechnic Institute”, 21 Frunze Str., Kharkov 61002, Ukraine

Received October 20, 2015, published online December 23, 2015

\begin{abstract}
Phonon and electron spectra of metallic bigraphene are analyzed in the presence of step-edge crystal imperfection. Different geometries of step-edge are considered. The dynamic planar stability of the considered structure is proved for temperatures above the ambient. The number of phonon states is shown to grow near the K-point of the first Brillouin zone, compared to pristine graphene. It is found, that this type of defects causes substantially nonuniform distribution of electron states and the pronounced increase in the number of states with energies close to Fermi energy can be expected in electron spectrum of the graphene-based compounds. The performed calculations are in good agreement with inelastic neutron, x-ray and Raman measurements.
\end{abstract}

PACS: 63.22.-m Phonons or vibrational states in low-dimensional structures and nanoscale materials;

73.22.-f Electronic structure of nanoscale materials and related systems;

73.22.Pr Electronic structure of graphene.

Keywords: quasiparticle spectrum, quasi-two-dimensional crystals, graphene, bigraphene.

\section{Introduction}

In the structures of coherently stacked graphene monolayers (graphite as the end family-member) a honeycomb layers packing is remained, but Dirac singularity in electron spectrum is lost due to weak van der Waals coupling of the layers. The latter gives rise to normal-metal conductivity in such systems instead of zero-gap semiconducting (semimetal) behavior of monolayer graphene [1,2]. A growing interest to these systems is caused by their direct relevance to the manufacturing of the advanced, closed nanoarrangements of carbon (see, e.g., [3-6]), and those of transition-metal dichalcogenides $[7,8]$.

The Dirac V-shape singularity of electron density of states (DOS) in the point of charge neutrality, coincident with Fermi energy $\varepsilon_{F}$ in graphene, makes the corresponding spectral characteristics and, eventually, transport properties highly sensitive to the type and content of crystal imperfections in graphene-based compounds [9-19]. The semiconducting [20]/superconducting $[21,22]$ gaps opening, for instance, is assumed. Hence, the present study explores, how the step-edges of different chirality affect electron spectrum, 
vibraional properties and their interplay in bigraphene, the simplest representative of such structures, which is frequently used as the model system. The quasiparticle local densities of states near the step-edge and their influence on spectral properties of Bernal-stacked bilayer graphene near $\varepsilon_{F}$ are discussed in comparison with inelastic neutron [9-11], x-ray [23] and Raman [5,10,24] scattering measurements. The numerical calculations are performed using the Jacobi matrix technique [24-27], which provides the full set of eigenfunctions without taking the translational symmetry into account. The force constants and phonon DOS are taken from papers $[8,14]$ as well as contribution of atomic displacements in principal crystallographic directions.

\section{The phonon spectra and mean square amplitudes of atomic vibrations near the step-edge of carbon nanofilm}

Similar to graphene monolayer, the honeycomb structure of basal layers in bigraphene is composed of the carbon close-packed triangles (Fig. 1(a)). These two graphene monolayers are coupled, similar to the bulk graphite, by van der Waals interaction. The interlayer spacing or film thickness is $h \sim 3.5 \AA$. In contrast to unit cell of graphite with two physically equivalent atoms, i.e., equal local Green functions and local densities of states, the unit cell of bigraphene consists of four atoms, with different interaction of atoms pertained to different sublattices in one layer with those pertained to another layer. Their physical equivalence is, therefore, destroyed (Fig. 1(a)), while the atoms of different layers are, naturally, equivalent. The corresponding Brillouin zone (BZ) with high-symmetry points is demonstrated in Fig. 1(b).

Obviously, the in-plane properties are not much affected by interlayer interaction in graphene. Rather the out-ofplane phonon displacements should be responsible for observed specific vibrational properties in bigraphene.

The step-edge in bigraphene originates from stackingmisfit boundary between the graphene layers of different size. Here the typical case of half-planes filled with bigraphene and graphene are considered. Possible configurations of step-edge atoms distribution are shown in Fig. 1(c)-(e). Though it was demonstrated before $[8,14]$ that bigraphene and graphene keep plane geometry at temperatures much above the ambient one, the stability limits of step-edge atoms with dangling bonds remains unexplored. For straightforward estimation of the stability limits for step-edge atoms, the structure of the type "trigraphene step-edge" can be chosen, when another graphene monolayer represents the substrate. It is absolutely clear, that real substrates of macroscopic thickness would provide even less values of mean-square amplitudes of atomic displacements and, eventually, the broader temperature ranges of structure stability.

Phonon spectrum and vibrational properties of the boundary are strongly dependent on its configuration. Those of two types, "armchair" (Fig. 1(c)) and "zig-zag" (Fig. 1(d)-(e)), are considered. For the latter, two cases are studied, either with a single dangling bond on the atom pertained to lattice $A(\bullet)$ (Fig. 1(d)), or that for sublattice $B$ (०) (Fig. 1(e)).

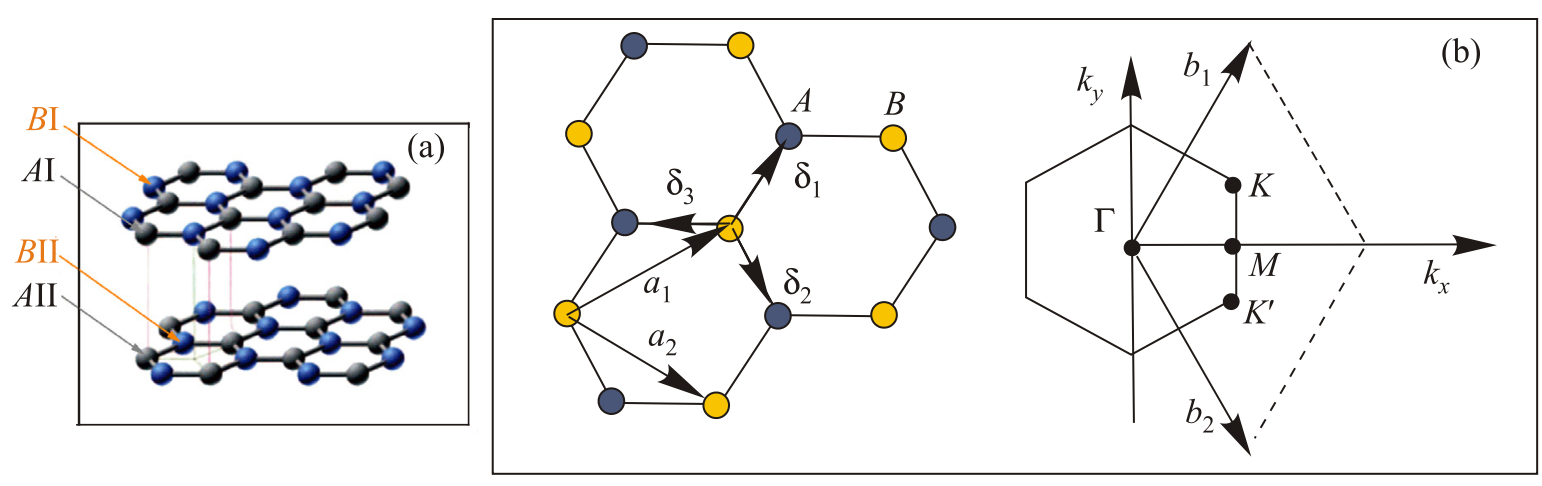

(c)

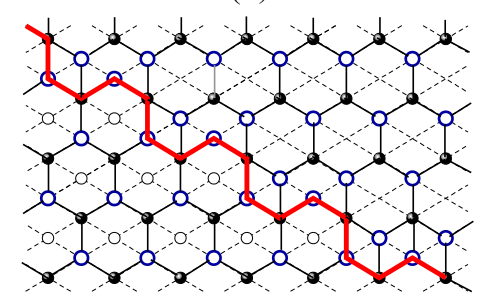

armchair (d)

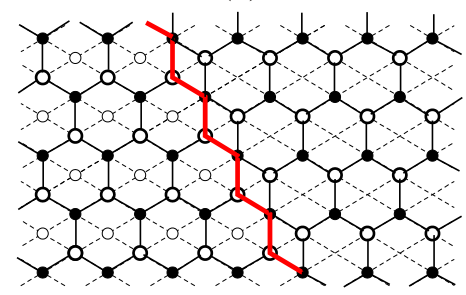

zig-zag $A$ (e)

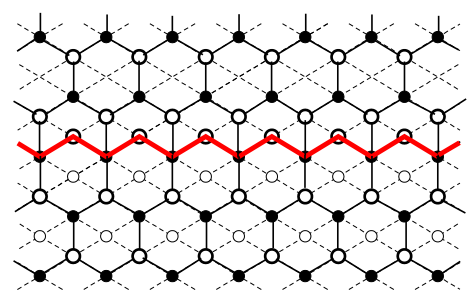

zig-zag $B$

Fig. 1. (Color online) Crystal structure (a), Brave lattice and first BZ of bigraphene (b); step-edge configurations under study (c)-(e). 
The phonon densities of state, originated from displacements in $c$-axis direction of the atoms pertained to both sublattices of bulk graphite and those of carbon nanofilms, are featured by a deep minimum at $\omega=\omega(K)$ (Fig. 2(a) green (3) and wine (2) dependences, respectively). This minimum resembles the Dirac V-singularity in electron density of grahene and similarly is responsible imperfection induced quasilocal states with frequencies close to $\omega(K)[14,28]$. Note, that namely the polarized normal to graphene layers (quasiflexural) vibrations [29] should play a crucial role in, say, the Cooper pairing at superconducting transition. In Fig. 2(a) blue curve 1 is the phonon spectral density, set by normal to the layer displacement of the boundary atom of step-edge zig-zag $A$. The quasilocal maximum is clearly seen in a frequency range slightly below $E(K) \equiv \hbar \omega(K) / e$ ( $e$ is the electron charge), as well as a pronounced growth of the number of phonon states at $E=E(K)$. It matches the observed phonon spectrum transformations at the superconducting transition in a number of metal-intercalated graphite
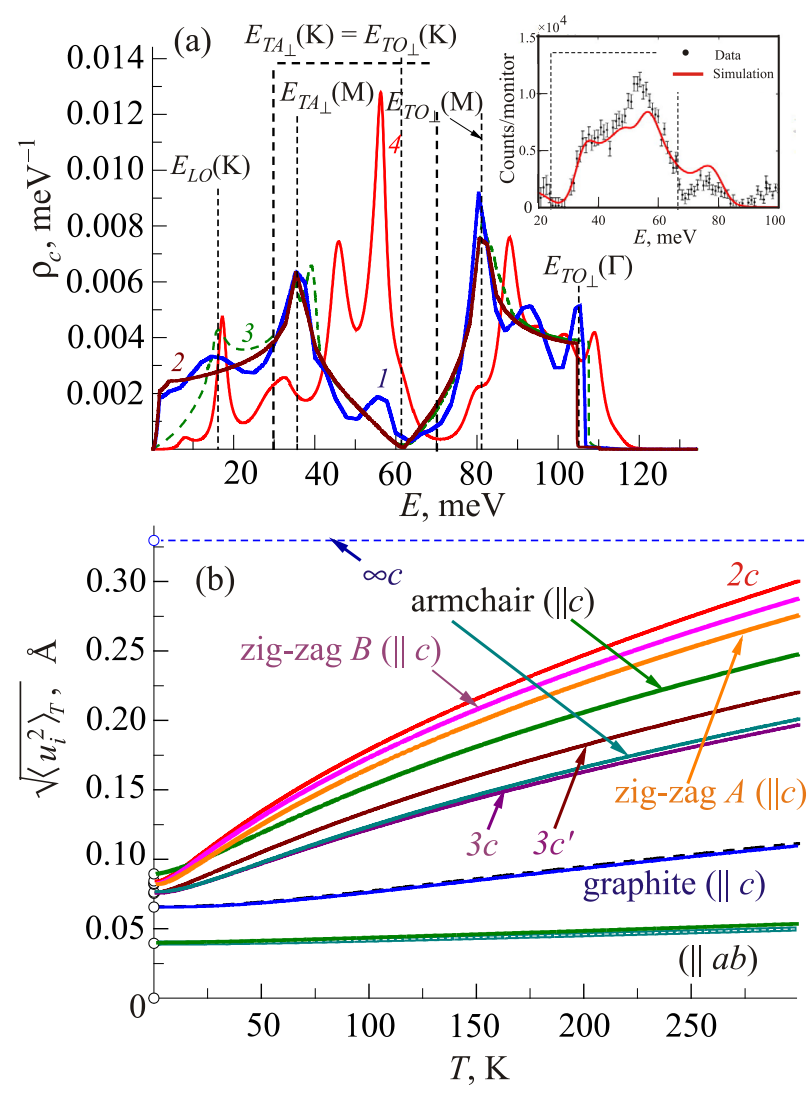

Fig. 2. (Color online) Spectral phonon properties originated by step-edge imperfection compared to graphene and its derivatives: (a) displacement along the $c$ axis of the atom in step-edge zig-zag $A$ (1); clean bigraphene (2), graphite (3), Ca-intercalated graphite [5,19] (4). Inset: symbols are neutron measurements of phonon densities for $\mathrm{CaC}_{6}$, solid line $a b$ simulations [21]. (b) RMS atomic displacements along different crystallographic directions in bigraphene, trigraphene and different configurations of a bigraphene-trigraphene interface. systems [9-13]. Thus, in paper [12], substantial increase of phonon number near $E(K)$ is evidenced by inelastic neutron measurements for $\mathrm{CaC}_{6}$-intercalated graphite. In [12], a perfect agreement of this result with $a b$ initio calculations [30,31] is demonstrated. Interestingly, the same result was derived [14,28] within a trivial lattice dynamics approach (red curve 4) notwithstanding the perfect crystal structure consideration which inevitably sharpers the resonances in spectral densities. The role of the number of phonon states at $E=E(K)$ is also proved by observations [12] and relevant calculations $[14,28]$ of the transition temperature $T_{c}$ decrease with weakening of intercalation effect on the phonon DOS of graphite near $E(K)$, e.g., $T_{c}=11.5 \mathrm{~K}$ for $\mathrm{CaC}_{6}$, compared to $6.5 \mathrm{~K}$ for $\mathrm{YbC}_{6}$. Note, that above considered measurements did not show much difference in occupancy growth near $\varepsilon_{F}$ at doping with $\mathrm{Ca}$ and $\mathrm{Yb}$. It is also worth noting here, that in graphite intercalated with lithium, which is more than twice lighter than carbon, $T_{c}=1.9 \mathrm{~K} \mathrm{[32].} \mathrm{The}$ reason is that the light atom vibrations weakly affect the phonon spectrum of graphite near $E=E(K)$, being rather concentrated in the higher-frequency region.

The temperature dependence of root-mean-square deviation (rmsd) displacements of atom $s$ in a crystallographic direction $i$ relates to corresponding spectral density as

$$
\left\langle\left[u_{i}^{(s)}\right]^{2}\right\rangle_{T}=\frac{\hbar}{2 m^{(s)}} \int_{D} \frac{\rho(\omega)}{\omega} \operatorname{cth}\left(\frac{\hbar \omega}{2 k_{B} T}\right) d \omega .
$$

Calculations of rmsd amplitudes of atomic displacements $\sqrt{\left\langle\left[u_{i}^{(s)}\right]^{2}\right\rangle_{T}}$ for atoms of free-stand, epitaxial bigraphene (the trigraphene here) and their interfaces of various configurations (Fig. 2(b)) yield a weak variation of rmsd in $a b$ plane without any significant difference from a bulk graphene nanofilm. At the same time rmsd along the $c$ axis show a pronounced growth with decreasing the number of layers (in Fig. 2(b): $2 c$ labels bigraphene; $3 c$ and $3 c^{\prime}$ edge and middle layers of trigraphene, respectively; $\infty c$ bulk graphite). The MSD of step atoms are seen to locate between curves $3 c$ and $2 c$ not approaching the meaning $\infty c(T=3000 \mathrm{~K})$ even at ambient temperatures (the melting temperature of graphite is $\sim 4000 \mathrm{~K}$ ). Therefore the step-edge in epitaxial bigraphene with the most rigid condition of the trigraphene-surface configuration meets the stability requirements for further investigation of electron spectrum. The bilayer graphene with steps of different chirality synthesized on Si-graphite is stable at much higher temperature (above $1000 \mathrm{~K}$ ) [33].

\section{The step-edge effect on electron spectrum of bigraphene}

\subsection{Electron spectrum of metallic bigraphene}

Electron spectrum of bigraphene is similar to that of grapheme and can be described within a tight-binding approximation. Hamiltonian based on the Wannier representation can be expressed as (see, e.g., [8]) 


$$
\hat{H}=\sum_{i} \varepsilon_{i}|i\rangle\left\langle i\left|-\sum_{i, j} J_{i j}\right| i\right\rangle\langle j|
$$

where $i$ and $j$ denote the neighboring sites on the lattice $\varepsilon_{i}$ is the on-site energy, and $J_{i j}$ is the nearest hopping energy. In assumption that, similar to graphene, the inlayer electron hopping is possible only for nearest neighbors $J_{i j}=J \approx 2.8-3.1 \mathrm{eV}$ (see, e.g., [33]). The interlayer electron hopping is also assumed possible only between the nearest neighbors from different layers, i.e., those separated by distance $h$. The relevant hopping integral is designated $J^{\prime}$. It should be noted, that such neighbors exist for half of bigraphene atoms, pertained to sublattices $A \mathrm{I}$ and $A$ II (see Fig. 1(a)). The atoms of sublattices $B \mathrm{I}$ and $B \mathrm{II}$ miss such the neighbors because their nearest neighbors from adjacent layers are set at the distance $\sqrt{h^{2}+a^{2}}$. Here $a \approx 1.415 \AA$ is the in-plane nearest neighbors separation. Though it exceeds $h$ for less than $10 \%$, the interaction with atoms of sublattices $B \mathrm{I}$ and $B \mathrm{II}$ is omitted, as it does not qualitatively affect the behavior of spectral characteristics near $\varepsilon_{F}$ (see, e.g., [5]).

The electron spectrum of graphene (see, e.g., $[1,2]$ ) is known to pertain two branches. The corresponding dispersion relations $\varepsilon_{0}(\mathbf{k})$ in tight-binding approximation (TBA) read

$$
\varepsilon_{0}(\mathbf{k})= \pm J \sqrt{1+4 \cos \left(\mathbf{k} \cdot \frac{\mathbf{a}_{1}-\mathbf{a}_{2}}{2}\right)\left[\cos \left(\mathbf{k} \cdot \frac{\mathbf{a}_{1}+\mathbf{a}_{2}}{2}\right)+\cos \left(\mathbf{k} \cdot \frac{\mathbf{a}_{1}-\mathbf{a}_{2}}{2}\right)\right]}
$$

where

$$
\mathbf{a}_{1}=\left(\frac{3 a}{2}, \frac{a \sqrt{3}}{2}, 0\right), \quad \mathbf{a}_{2}=\left(\frac{3 a}{2},-\frac{a \sqrt{3}}{2}, 0\right)
$$

are the two-dimensional lattice vectors and $a$ being the nearest-neighbor separation (see Fig. 1(b)). In (3), the energy is counted from the reference Fermi level and vanishes only in the K-points of the first BZ:

$$
\mathrm{K}=\left(0, \pm \frac{4 \pi}{3 a \sqrt{3}}, 0\right) \cup\left( \pm \frac{2 \pi}{3 a}, \pm \frac{2 \pi}{3 a \sqrt{3}}, 0\right) \cup\left( \pm \frac{2 \pi}{3 a}, \mp \frac{2 \pi}{3 a \sqrt{3}}, 0\right) .
$$

Four atoms in the elementary cell of bigraphene yield dispersion relations with four branches of its electron spectrum which are written in TBA as

$$
\begin{aligned}
& \varepsilon_{1,2}(\mathbf{k})= \pm \sqrt{\varepsilon_{0}^{2}(\mathbf{k})+\frac{J^{\prime 2}}{2}-J^{\prime} \sqrt{\varepsilon_{0}^{2}(\mathbf{k})+\frac{J^{\prime 2}}{4}}}, \\
& \varepsilon_{3,4}(\mathbf{k})= \pm \sqrt{\varepsilon_{0}^{2}(\mathbf{k})+\frac{J^{\prime 2}}{2}+J^{\prime} \sqrt{\varepsilon_{0}^{2}(\mathbf{k})+\frac{J^{\prime 2}}{4}}},
\end{aligned}
$$

where the $\varepsilon_{0}(\mathbf{k})$ function is given by Eq. (3). In the K-point of first BZ, the Brillouin function $\varepsilon_{1,2}(\mathbf{k})$ vanish, in contrast to function $\varepsilon_{3,4}(\mathbf{k})$, which means that in these modes Fermi level takes place within the energy gap.

The dispersion curves of bigraphene along the highsymmetry directions $\Gamma \mathrm{K}, \Gamma \mathrm{M}$ and $\mathrm{KM}$ for the case of $J^{\prime}=0.1 J$ are illustrated by Fig. 3(a). In the figure, there are pointed out the energy values in high-symmetry points $\Gamma, \mathrm{M}$ and $\mathrm{K}$ of the first $\mathrm{BZ}$ (for their location see Fig. 1(b)):

$$
\begin{gathered}
\varepsilon_{1,2}(\mathrm{~K})=0 ; \quad \varepsilon_{3,4}(\mathrm{~K})= \pm J^{\prime} \\
\varepsilon_{1,2}(\mathrm{M})= \pm \sqrt{J^{2}+J^{\prime 2} / 2-J^{\prime} \sqrt{J^{2}+J^{\prime 2} / 4}} \\
\varepsilon_{3,4}(\mathrm{M})= \pm \sqrt{J^{2}+J^{\prime 2} / 2+J^{\prime} \sqrt{J^{2}+J^{\prime 2} / 4}} \\
\varepsilon_{1,2}(\Gamma)= \pm \sqrt{9 J^{2}+J^{\prime 2} / 2-J^{\prime} \sqrt{9 J^{2}+J^{\prime 2} / 4}} \\
\varepsilon_{3,4}(\Gamma)= \pm \sqrt{9 J^{2}+J^{\prime 2} / 2+J^{\prime} \sqrt{9 J^{2}+J^{\prime 2} / 4}} .
\end{gathered}
$$

In the inset of Fig. 3(a), the K-point area is shown at magnified scale. The same inset demonstrates the dispersion relations of graphene (3). The quasirelativistic character of electron spectrum of graphene is clearly seen, as well as the «common» square-law run of the dispersion curve $\varepsilon_{1,2}(\mathbf{k})$ close to K-point. The spectral branches $\varepsilon_{3,4}(\mathbf{k}) \notin\left(-J^{\prime}, J^{\prime}\right)$.

The dispersion relations (4) are in good agreement with the Raman measurements.

In fact, for $\mathbf{k} \in \Gamma \mathrm{K}, \varepsilon_{0}(k)= \pm(1+2 \cos (a k \sqrt{3} / 2))$ and, if $\mathbf{k}=\mathbf{K}+\mathbf{K}$ ( $\mathbf{K}$ is the radius-vector of $K$-point in $\mathbf{k}$ space, $\kappa<<1)$, then $\varepsilon_{0}(\mathbf{K}+\mathbf{\kappa}) \approx \mp 3 a \kappa / 2$, is obtained which follows the linear (relativistic) dispersion law. The electron modes of bigraphene $\varepsilon_{1,2}(\mathbf{k})$ near the K-point with $\varepsilon_{0}(k)<<J^{\prime}$ are written as

$$
\begin{gathered}
\varepsilon_{1,2}^{2}(\mathbf{K}+\mathbf{\kappa}) \approx \frac{J_{1}^{2}}{2}+\varepsilon_{0}^{2}(\mathbf{K}+\mathbf{\kappa})-\sqrt{\left(\frac{J_{1}^{2}}{2}+\varepsilon_{0}^{2}(\mathbf{K}+\mathbf{K})\right)^{2}-\varepsilon_{0}^{4}(\mathbf{K}+\mathbf{K})} \approx \frac{J_{1}^{2}}{2}+\varepsilon_{0}^{2}(\mathbf{K}+\mathbf{\kappa})- \\
-\left[\frac{J_{1}^{2}}{2}+\varepsilon_{0}^{2}(\mathbf{K}+\mathbf{K})\right]\left\{1-\frac{\varepsilon_{0}^{4}(\mathbf{K}+\mathbf{K})}{2\left[J_{1}^{2} / 2+\varepsilon_{0}^{2}(\mathbf{K}+\mathbf{K})\right]^{2}}\right\}=\frac{\varepsilon_{0}^{4}(\mathbf{K}+\mathbf{\kappa})}{J_{1}^{2}+2 \varepsilon_{0}^{2}(\mathbf{K}+\mathbf{\kappa})} \approx \frac{81 J^{4} a^{4} \kappa^{4}}{16 J_{1}^{2}},
\end{gathered}
$$




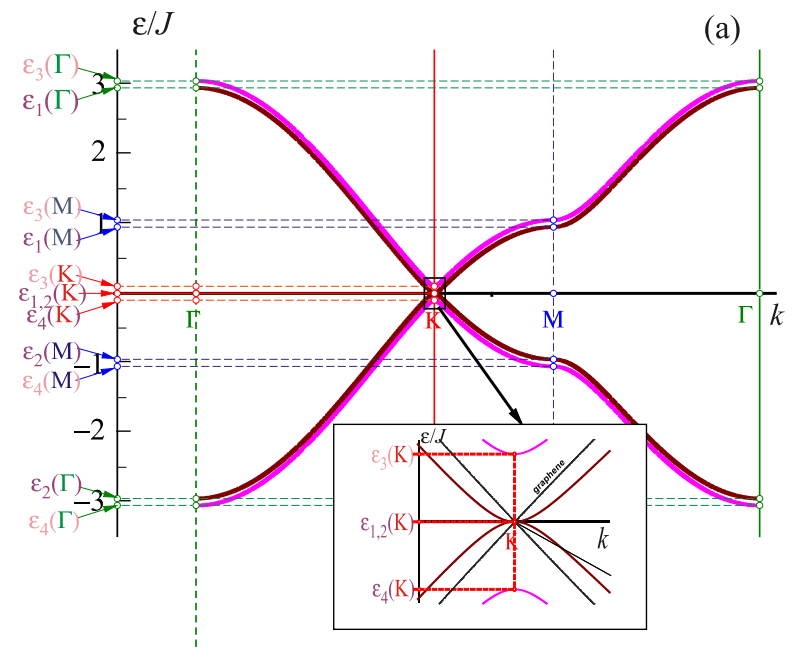

(b)

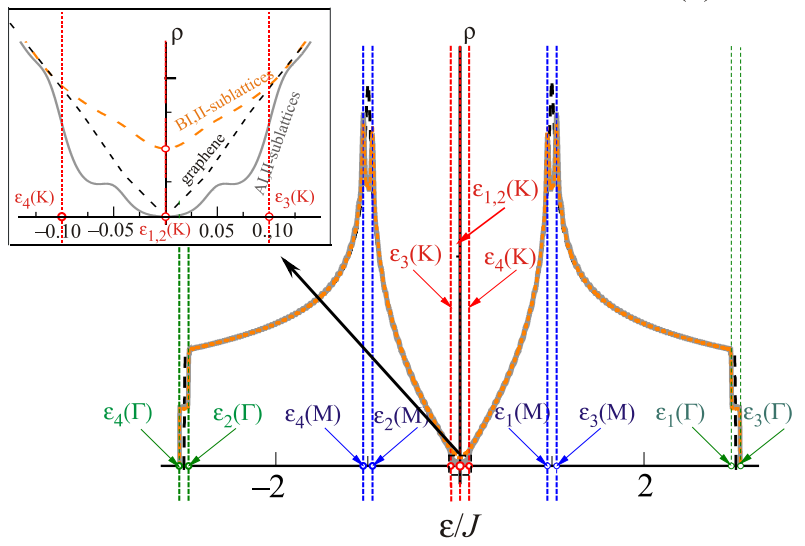

Fig. 3. (Color online) Dispersion of bigraphene: (a) along the high-symmetry directions. Inset: magnified area near the K-point of the first BZ, the dashed line is the graphene. (b) Local DOS of atoms pertained to diferrent lattices of bigraphene. Inset: magnified area around $\varepsilon_{i}(\mathrm{~K})$.

the trivial square law of dispersion is thus obtained

$$
\varepsilon_{1,2}(\mathbf{K}+\mathbf{\kappa}) \approx \pm\left[\frac{9 J^{2} a^{2} \kappa^{2}}{4 J_{1}}-o\left(\kappa^{2}\right)\right]
$$

The effective electron mass, derived from equation $\varepsilon=(\hbar \kappa)^{2} / 2 m^{*}$ for considered branches, appears to be equal to

$$
m^{*}=\frac{2 \hbar^{2} J^{\prime}}{9 J^{2} a^{2}} .
$$

For the considered case of $J^{\prime}=0.1 J$, the effective electron mass is $m^{*} \approx 2.75 \cdot 10^{-32} \mathrm{~kg}$, at $J^{\prime} \rightarrow J$ it approaches the value close to the order of magnitude of the free electron mass $m_{e} \approx 9 \cdot 10^{-31} \mathrm{~kg}$. As $m^{*} \sim J^{\prime}$, the interlayer hopping integral variation, e.g., by strain, allows one to change effective mass of charge carriers by the orders of magnitude. It should be noted then, that notwithstanding $J^{\prime} \rightarrow 0$ at such the boundary transition from bigraphene to two noninteracting graphene monolayers and the effective mass $m^{*} \rightarrow 0$, the Eqs. (7) and (8) become invalid here as obtained in assumption of $\varepsilon_{0}(k)<<J^{\prime}$.

Electron density of states (DOS) at energies close to $\varepsilon_{F}$ is determined by the branches $\varepsilon_{1}$ and $\varepsilon_{2}$ only (electron modes $\varepsilon_{3}$ and $\varepsilon_{4}$, reveal a gap in this range), and (4) yields $g_{1}(\varepsilon)=g_{2}(-\varepsilon)$. Then

$$
g(\varepsilon)=\frac{\Sigma_{0}}{(2 \pi)^{2}} \oint_{\varepsilon(\mathbf{\kappa})=\varepsilon} \frac{d l_{1,2}}{\left|\partial \varepsilon_{1,2} / \partial \mathbf{\kappa}\right|}
$$

where $\Sigma_{0}=3 a^{2} \sqrt{3} / 2$ is the square of the planar unit cell. Integration is performed over the closed isoenergetic line $\varepsilon(\kappa)=\varepsilon$. At $\varepsilon=0$ (Fermi level) this line shrinks to point and near $\varepsilon_{F}$ to the circumferential contour of integration. Using (8), we can write

$$
g_{1,2}\left(\varepsilon_{F}\right)=\lim _{\varepsilon \rightarrow \varepsilon_{F}} \frac{\Sigma_{0}}{(2 \pi)^{2}} \int_{0}^{2 \pi} \frac{\kappa d \varphi}{\left|\partial \varepsilon_{1,2} / \partial \kappa\right|}=\frac{J^{\prime}}{2 \pi J^{2} \sqrt{3}}=\text { const } .
$$

It means that at $\varepsilon=0$ DOS has a finite constant value. Moreover, according to (9), near the Fermi energy DOS is analytical function with minimum at $\varepsilon=0$ approaching $g(\varepsilon) \sim \varepsilon^{2}$ at $\varepsilon_{F}$.

The integral electron DOS can be described by the arithmetic mean value of two local densities of states (LDOS) $\rho_{S}(\varepsilon)$, apt to atoms from sublattices $A$ and $B$ (which follows from the above mentioned physical equivalency of atoms pertained to different bigraphene layers) $\rho_{A \mathrm{I}}(\varepsilon)=\rho_{A \mathrm{II}}(\varepsilon) \equiv \rho_{A}(\varepsilon) ; \quad \rho_{B \mathrm{I}}(\varepsilon)=\rho_{B \mathrm{II}}(\varepsilon) \equiv \rho_{B}(\varepsilon)$; $g(\varepsilon)=\left[\rho_{A}(\varepsilon)+\rho_{B}(\varepsilon)\right] / 2$. The LDOS for each of ideal sublattices can be represented as

$$
\rho_{S}\left(\varepsilon_{F}\right) \approx \frac{\Sigma_{0}}{(2 \pi)^{2}} \sum_{\alpha=1}^{q} \oint_{\varepsilon(\mathbf{k})=\varepsilon} \frac{\left|\psi_{S}(\alpha, \mathbf{k})\right|^{2}}{\left|\nabla_{\boldsymbol{k}} \varepsilon_{\alpha}(\mathbf{k})\right|} d l_{\alpha} .
$$

In (11) subscripts $s$ and $\alpha$ refer to sublattices and branches, respectively, $\psi_{S}(\alpha, \mathbf{k})$ are mean eigenfunctions of atoms from each sublattice. The LDOSs, calculated by Jacoby matrix technique [25-27] for each of the bigraphene sublattices, are illustrated by Fig. 3(b). The two-dimensional van Hove singularities at energy values corresponding to $\Gamma$ - and M-points of the first BZ are clearly seen. The inset presents the same dependences at magnified scale for energy range in the vicinity of $\varepsilon_{F}$. It shows, that near Fermi level, LDOS, as well as integral DOS, are analytical and their dependence on energy is sufficiently nonlinear (in contrast to DOS of graphene presented for a comparison in the inset). Moreover, the LDOS $\rho_{A}(\varepsilon)$ contrary to $\rho_{B}(\varepsilon)$ and integral DOS vanishes at $\varepsilon \rightarrow 0$. It is worth noting that near $\varepsilon=0$, the dependence $\rho_{A}(\varepsilon)$ is very smooth, much smoother than $\rho_{B}(\varepsilon)$, and the energy spectrum behavior for atoms of sublattice is a gap-like one. 
It means, that electron energy spectrum of sublattices $B \mathrm{I}$ and $B \mathrm{II}$ has a metallic character with effective mass of charge carriers (8), and that of sublattices $A \mathrm{I}$ and $A \mathrm{II}$ are the semiconducting one of finite, though small width.

Indeed, the substitution of zero eigenvalue in the equation for eigenfunctions of Hamiltonian (1), yields $\psi_{A \mathrm{I}} \sim \psi_{A \mathrm{II}}=O\left(\kappa^{2}\right)$, and $\psi_{B \mathrm{I}} \sim \psi_{B \mathrm{II}} \sim 1$. Then at the Fermi level $\rho_{A}(\varepsilon) \sim \varepsilon^{2} \rho_{B}(\varepsilon)$. The features in both of the LDOS at $\varepsilon \approx \pm 0.1 J= \pm J^{\prime}$ are attributed to contributions of the modes $\varepsilon_{3,4}$. For the latter, the interval $\varepsilon \in\left[-J^{\prime}, J^{\prime}\right]$ is the gap, and the features at $\varepsilon \approx \pm 0.05 J$ are caused by anisotropy of the isofrequency lines, which becomes noticeable starting just at these energy values.

\subsection{The electron LDOS for atoms around step-edges of different chiralities}

It was shown earlier $[5,11]$ that imperfections favor the occurrence of quasilocal states in electron spectra of carbon nanofilm. The presence of vacancies in graphite causes the appearance of sharp resonance in electron density of states near $\varepsilon=\varepsilon_{F}$, and in [19] the similar effect was predicted for bigraphene. Similar to vacancy, the considered imperfection is due to breaking the atomic bonds, and the same effect on electron spectrum can be expected here, at least for particular configurations.
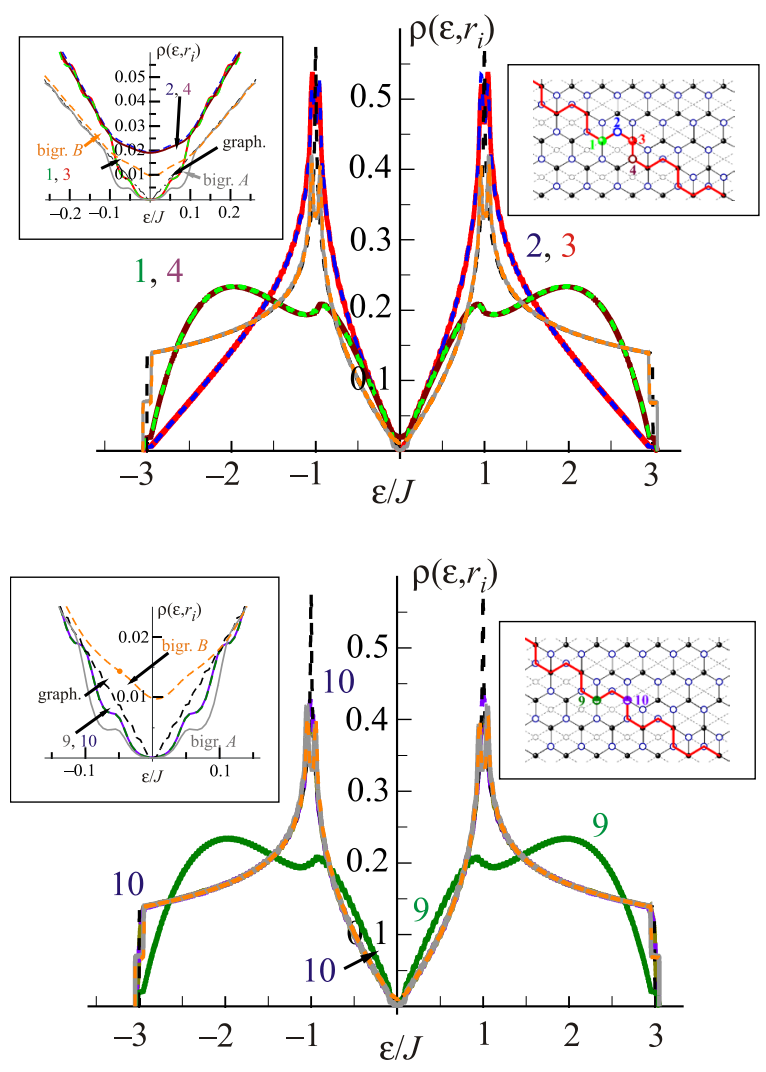

The Fig. 4 presents electron LDOS for the atoms, located near bigraphene step-edge of the armchair type. It is seen, that the most pronounced LDOS transformations occur for the atoms located in the plane of the edge (layer 1 on the top plane step). LDOS of the atoms on the bottom plane approach rather rapidly that of graphene or bigraphene, from the either occupied side of the step. In the case of armchair no of the LDOS reveal resonances near $\varepsilon=\varepsilon_{F}$. The LDOS behavior near $\varepsilon=\varepsilon_{F}$ for atoms of sublattice $B$, located at the boundary (upper left fragment, brown and blue curves), indicates higher, than for clean bigraphene (orange curve in inset), value of effective electron mass.

Even the more intriguing behavior is observed for LDOS of atoms, located near the step-edge zig-zag $A$, presented by Fig. 5. The LDOS of atoms from sublattices $A$ I (with a single dangling bond due to step formation), of those from $B$ II reveal sharp resonances at $\varepsilon=\varepsilon_{F}$ (the heights of atoms $A \mathrm{I}$, for several orders of magnitude exceed those of $B \mathrm{II})$. The maximum heights are decreased quite slowly with going away from the boundary inside bigraphene, and disappear immediately at the «graphene» side of step-edge (layer II). At the bigraphene side of step the Fermi-level of atoms belonging to sublattices $A \mathrm{II}$ and $B \mathrm{I}$ appears in the gap of width $\sim J^{\prime}$. At the «graphene» side of step-edge, the LDOS
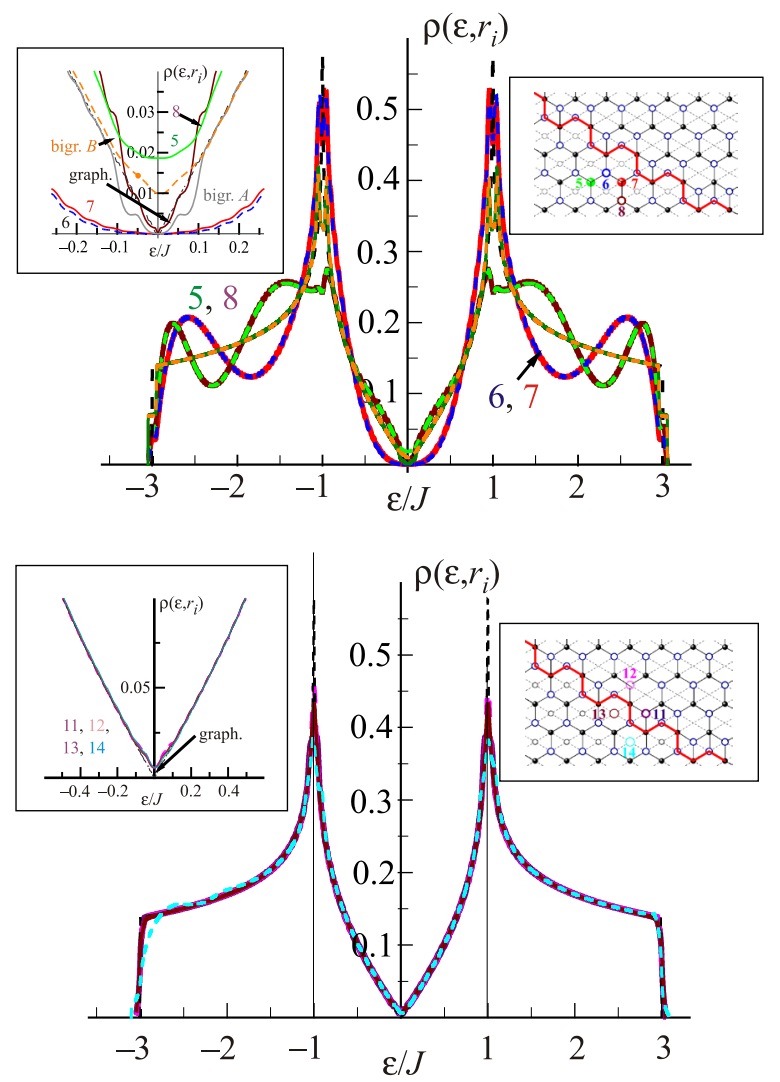

Fig. 4. (Color online) Atomic LDOS for the armchair step at the surface of bigraphene. The upper right part of each panel demonstrates the corresponding atoms distribution nearby step-edge (labels and color of the atoms are the same for their LDOS); at the left-hand the magnified portions of corresponding LDOS are presented near $\varepsilon=\varepsilon_{F}$. 

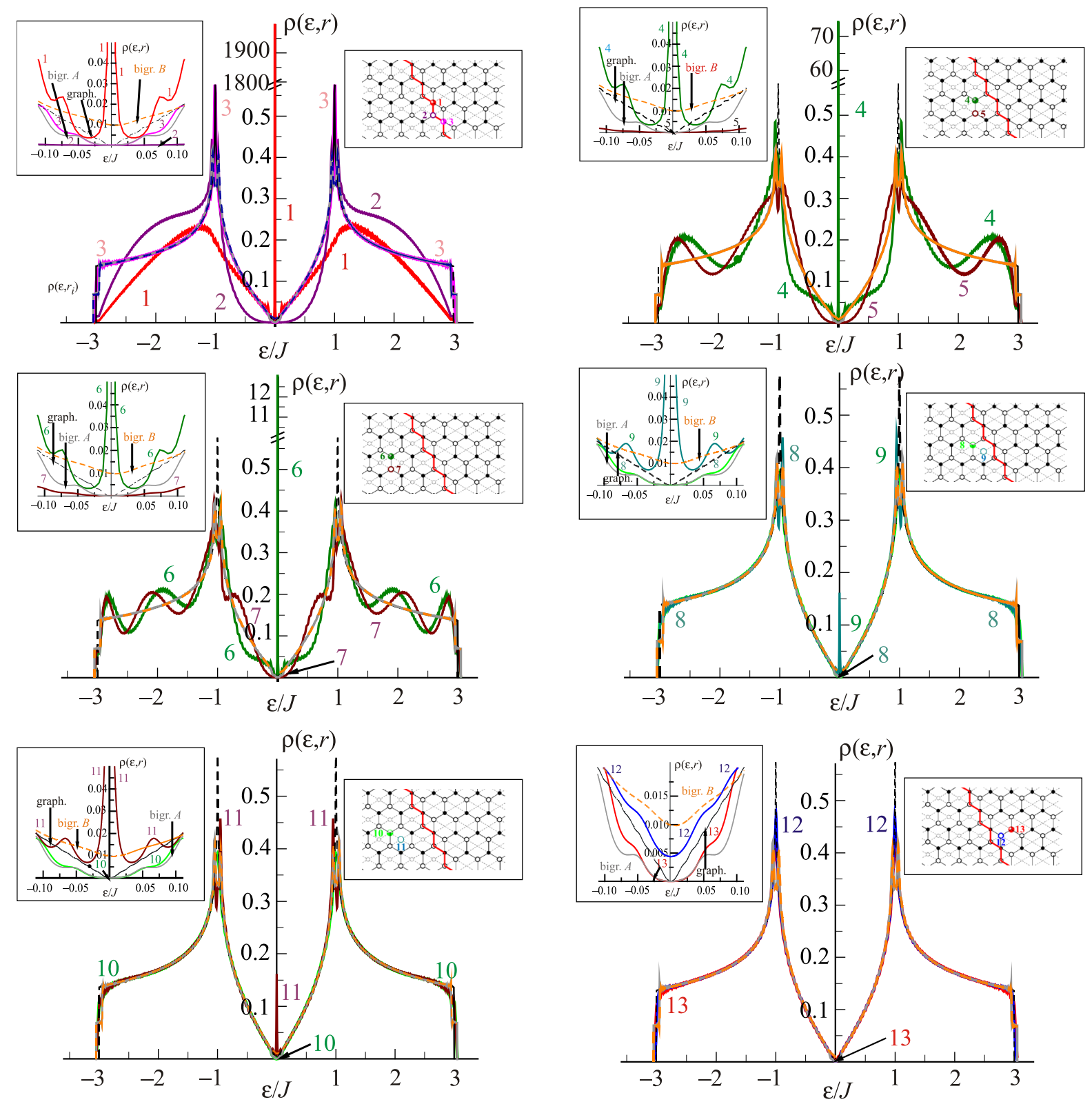

Fig. 5. (Color online) Atomic LDOS for the zig-zag step at the surface of bigraphene $A$ (the same designations as in Fig. 4).

correspond to those of ideal bigraphene, but their tendency towards LDOS of graphene is manifested immediately apart from the step-edge. The same behavior is manifested by LDOS in the case of step-edge zig-zag $B$. Similar to the previos case, the somewhat weaker resonance should be found in $\operatorname{LDOS} \rho_{A \mathrm{I}}(\varepsilon)$ and $\rho_{B \mathrm{II}}(\varepsilon)$, though in this case, the bonds are broken on atoms $B \mathrm{I}$.

\section{Conclusions}

Each of the bigraphene layers is considered as 2D superstructure arranged by metallic and semiconducting triangular lattices.

The numerical calculations compared with spectral measurements have shown that i) Spatial distribution of electron states on the step atoms with dangling bonds and their neighbors is nonuniform and depends on step-edge chirality.

ii) The integral DOS of clean bigraphene and that with step-edge differ near Fermi level, mainly: semiconducting gap opens for armchair configuration and the density of state increases for the zig-zag.

iii) The phonon spectral density grows at $\omega=\omega(K)$ for armchair, similar to interstitials, which facilitate a rather high-temperature superconductivity. The dynamical planar stability still remains up to temperatures about 300-400 K.

We thank G.E. Grechnev and K.A. Chishko for useful discussions. 
1. P.R. Wallace, Phys. Rev. 71, 622 (1947).

2. K.S. Novoselov, A.K. Geim, S.V. Morozov, D. Jiang, M.I. Katsnelson, I.V. Grigorieva, S.V. Dubonos, and A.A. Firsov, Nature 438, 197 (2005).

3. M.S. Dresselhaus and P.C. Eklund, Adv. Phys. 49, 705 (2000).

4. T. Ando, J. Phys. Soc. Jpn 74, 777 (2005).

5. Carbon Nanotubes, A. Jorio, G. Dresselhaus, and M.S. Dresselhaus (eds.), Topics Appl. Physics 111, SpringerVerlag, Berlin, Heidelberg (2008).

6. T.A. Len, L.Yu. Matzui, I.V. Ovsienko, Yu.I. Prylutskyy, V.V. Andrievskii, I.B. Berkutov, G.E. Grechnev, and Yu.A. Kolesnichenko, Fiz. Nizk. Temp. 37, 1027 (2011) [Low Temp. Phys. 37, 819 (2011)].

7. E. Bartolomé, J. Bartolomé, A. Arauzo, V.V. Eremenko, and V.A. Sirenko, J. Phys.: Condensed Matter 22, 295702 (2010).

8. I.A. Gospodarev, V.V. Eremenko, K.V. Kravchenko, V.A. Sirenko, E.S. Syrkin, and S.B. Feodosyev, Fiz. Nizk. Temp. 36, 436 (2010) [Low Temp. Phys. 36, 344 (2010)].

9. T.E. Weller, M. Ellerby. S.S. Saxena, R.P. Smith, and N.T. Skipper, Nat. Phys. 1, 39 (2005).

10. M.P.M. Dean, C.A. Howard, S.S. Saxena, and M. Ellerby, Phys. Rev. B 81, 045405 (2010).

11. M.Y. Upton, T.R. Forrest, A.C. Walters, C.A. Howard, M. Ellerby, A.H Said, and D.F. McMorrow, Phys. Rev. B 82, 134515 (2010).

12. M.P.M. Dean, A.C. Walters, C.A. Howard, T.E. Weller, M. Calandra, F. Mauri, M. Ellerby, S.S. Saxena, A. Ivanov, and D.F. McMorrow, Phys. Rev. B 82, 014533 (2010).

13. M.H. Upton, A.C. Walters, C.A. Howard, K.C. Rahnejat, M. Ellerby, J.P. Hill, D.F. McMorrow, A. Alatas, Bogdan M. Leu, and Wei Ku, Phys. Rev. B 76, 220501 (2007).

14. A. Feher, I.A. Gospodarev, V.I. Grishaev, K.V. Kravchenko, E.V. Manzhelii, E.S. Syrkin, and S.B. Feodosyev, Fiz. Nizk. Temp. 35, 862 (2009) [Low Temp. Phys. 35, 679 (2009)].

15. S. Pisana, M. Lazzeri, C. Casiraghi, K.S. Novoselov, A.K. Geim, A.C. Ferrari, and F. Mauri, Nature Mater. 6, 198 (2007).

16. A. Das, S. Pisana, B. Chakraborti, S. Piscane, S.K. Sahai, U.V. Waghmare, K.S. Novoselov, H.R. Krishnamurthy, A.K. Geim, A.C. Ferrari, and A.K. Sood, Nat. Nanotechnol. 3, 210 (2008).

17. M. Lazzeri and F. Mauri, Phys. Real. Lett. 97, 266407 (2006).

18. J.C. Tsang, M. Freiting, V. Perebeinos, J. Liu, and P. Avouris, Nat. Nanotechnol. 2, 725 (2007).
19. A. Feher, E. Syrkin, S. Feodosyev, I. Gospodarev, and K. Kravchenko, Quasi-Particle Spectra on Substrate and Embedded Graphene Monolayers, in: Physics and Application of Graphene - Theory, S. Mikhailov (ed.), InTex Open Access Publisher, ISBN 978-953-307-996-7 Croatia (2010).

20. Yu. Zhang, T.-T. Tang, C. Girit, Zh. Hao, M.C. Martin, A. Zettl, M.F. Crommie, Y. Ron Shen, and F. Wang, Nature Lett. 459, 820 (2009).

21. G. Savini, A.C. Ferrari, and F. Giustino, Phys. Rev. Lett. 105, 037002 (2010).

22. V.M. Loktev and V. Turkowski, J. Low Temp. Phys. 164, 264 (2011).

23. J. Maultzsch, S. Reich, C. Thomsen, and H. Requardt, Phys. Rev. Lett. 92, 075501 (2004).

24. A.C. Ferrari, J.C. Meyer, V. Scardaci, C. Casiraghi, M. Lazzeri, F. Mauri, S. Piscanec, D. Jiang, K.S. Novoselov, S. Roth, and A.K. Geim, Phys. Rev. 97, 187401 (2006).

25. V.I. Peresada, in: Condensed Matter Physics (in Russian), FTINT AN UkrSSR, Kharkov (1968), p. 172.

26. V.I. Peresada, V.N. Afanasyev, and V.S. Borovikov, Fiz. Nizk. Temp. 1, 461 (1975) [Sov. J. Low Temp. Phys. 1, 227 (1975)].

27. R. Haydock, in: Solid State Physics 35, H. Ehrenreich et al. (eds.), Academic Press, New York (1980), p. 129.

28. A. Feher, E. Syrkin, S. Feodosyev, I. Gospodarev, E. Manzhelii, A. Kotlyar, and K. Kravchenko, Electronic and Vibrational Properties of Adsorbed and Embedded Graphene and Bigraphene with Defects, in: New Progress on Graphene Research, Jian Ru Gong (ed.): InTech, ISBN 978-953-51-1091-0 Croatia (2013).

29. E.G. Maksimov, Usp. Phys. Nauk. 178, 175 (2008) [Phys. Usp. 51, 167 (2008)].

30. M. Calandra and F. Mauri, Phys. Rev. Lett. 95, 237002 (2005).

31. J.S. Kim, L. Boeri, R.K. Kremer, and F.S. Razavi, Phys. Rev. $B$ 74, 214513 (2006).

32. I.T. Belash, A.D. Bronnikov, O.V. Zharikov, and A.V. Pal'nichenko, Solid State Commun. 69, 921 (1989).

33. M.H. Oliveira, J.M.J. Lopes, T. Schumann, L.A. Galves, M. Ramsteiner, K. Berlin, A. Trampert, and H. Riechert, Nature Commun. 6, 7632 (2015).

34. K.S. Novoselov, Usp. Fiz. Nauk 181, 1299 (2011). 